\title{
Estimation of tumor microvessel density by MRI using a blood pool contrast agent
}

\author{
FUMINORI HYODO $^{1,2}$, GADISETTI V.R. CHANDRAMOULI ${ }^{1}$, SHINGO MATSUMOTO ${ }^{1}$, \\ KEN-ICHIRO MATSUMOTO ${ }^{3}$, JAMES B. MITCHELL ${ }^{1}$, MURALI C. KRISHNA ${ }^{1}$ and JEEVA P. MUNASINGHE ${ }^{4}$ \\ ${ }^{1}$ Radiation Biology Branch, Center for Cancer Research, National Cancer Institute, NIH, Bethesda, MD, USA; \\ ${ }^{2}$ Innovation Center for Medical Redox Navigation, Kyushu University, Fukuoka; \\ ${ }^{3}$ Heavy-Ion Radiobiology Research Group, National Institute of Radiological Sciences, Chiba, Japan; \\ ${ }^{4}$ National Institute of Neurological Disorders and Stroke, NIH, Bethesda, MD, USA
}

Received June 4, 2009; Accepted July 2, 2009

DOI: 10.3892/ijo_00000392

\begin{abstract}
Recognition of importance of angiogenesis to tumor growth, metastasis, and treatment outcome has led to efforts to develop non-invasive methods for longitudinal monitoring of tumor microvasculature. We describe a steadystate MRI technique to determine absolute blood volume (BV) as a marker of microvascular density with improved spatial and temporal resolution using an ultra small super paramagnetic iron oxide (USPIO). A noise reduction scheme for BV imaging was also proposed based on weighting factors derived by pre-contrast signal level as an adjustable additive constant. Gradient echo sequence was used for BV imaging with MRI at 7T. Optimal imaging conditions (USPIO dose and echo time) were determined by USPIO dose-dependent studies ex vivo and in vivo. Improved analysis strategies were at first applied for cerebral BV estimation in mice, which were found in good agreement with the literature values. These methods were then used to determine tumor $\mathrm{BV}$ in mice. The optimal concentration of USPIO for BV estimates was found to range from 3.6 to $4.48 \mathrm{mM}$ (estimated as $\mathrm{Fe}$ concentration) in ex vivo experiments corresponding to an in vivo dosage of $215-287 \mu \mathrm{mol} / \mathrm{kg}$ body weight, whereas a USPIO dose of $287 \mu \mathrm{mol} / \mathrm{kg}$ leads to higher cerebral BV estimate in vivo than the reported values. Application of the $\mathrm{BV}$ imaging method to evaluation of anti-angiogenic effect of Sunitinib in squamous cell carcinoma (SCC) tumor bearing mice revealed $\sim 46 \%$ reduction in tumor BV 4 days after start of Sunitinib treatment. The results show that the MRI approach using USPIO yields high-resolution absolute BV images and the method can be conveniently applied to monitor
\end{abstract}

Correspondence to: Dr Murali C. Krishna, Radiation Biology Branch, Center for Cancer Research, National Cancer Institute, NIH, Building 10, Room B3B69, Bethesda, MD 20892, USA E-mail: murali@helix.nih.gov

Key words: angiogenesis, blood volume, microvascular density, MRI, Sunitinib, tumor, USPIO longitudinal tumor microvessel density changes as a function of growth or in response to treatment.

\section{Introduction}

Solid tumors are characterized by promoted angiogenesis and resultant structural and functional abnormality of neovasculature $(1,2)$. The lack of adequate pericyte and endothelial coverage results in large vascular pores causing a marked regional heterogeneity in tumor blood perfusion and limited supply of oxygen, glucose, and other nutrients (2-4). In addition, recent treatment strategies using anti-angiogenic drugs have shown that tumor vessels are transiently normalized by pruning immature vessels to make the remaining vessels functionally more efficient (5). Functional improvement of the remaining blood vessels has been attributed to microvascular maturation via improved perivascular cell coverage and decreased vascular permeability. Consequently the increase in blood perfusion and decrease in hypoxic faction improved treatment response to radiotherapy and also enhanced chemotherapeutic drug delivery to an actively dividing cell population $(6,7)$.

Current advances in blood perfusion imaging have made it possible to quantify the number and spacing of blood vessels, measure blood flow and vascular permeability, and analyze cellular and molecular abnormalities in blood vessel walls. Microscopic imaging tools are used for dissecting the cellular and molecular features of microvasculature. MRI, PET, CT and other non-invasive imaging methods can localize sites of tumor angiogenesis in animals and patients. Tumor blood volume (BV), which is directly related to microvessel density, is non-invasively available indicator of angiogenesis. Assessment of BV by MRI using various contrast agents has been described in several studies (8-16).

Ultra small super paramagnetic iron oxide (USPIO) is a steady state blood pool agent that creates contrast through magnetic susceptibility variations proximal to the vascular network $(8,10,14,15)$, which results in changes in inherent and induced transverse relaxation times defined as, respectively, $T_{2}$ and $T_{2}{ }^{*}$. Accurate evaluation of $R_{2}{ }^{*}\left(1 / T_{2}{ }^{*}\right)$ or $R_{2}\left(1 / T_{2}\right)$ necessitate defining two or more points on the natural or 
induced transverse relaxation decay curve. Addition of contrast agent increases $\mathrm{R}_{2}{ }^{*}\left(1 / \mathrm{T}_{2}{ }^{*}\right)$ thus causing limits on sampling as the $\mathrm{T}_{2}{ }^{*}$ is shortened. It can be shown that:

$$
\Delta \mathrm{R}_{2}{ }^{*} \text { or } \Delta \mathrm{R}_{2} \propto \log \left[\frac{\mathrm{S}^{\text {post }}}{\mathrm{S}^{\text {pre }}}\right]
$$

where, $S^{\text {pre }}$ and $S^{\text {post }}$ are pre- and post-contrast MRI signal intensity. The changes in transverse relaxation rate $\Delta R_{2}\left(1 / \Delta T_{2}\right)$ and changes in effective transverse relaxation rate $\Delta R_{2}{ }^{*}$ $\left(1 / \Delta \mathrm{T}_{2}{ }^{*}\right)$ caused by the contrast agent are proportional to $\mathrm{BV}$ fraction (9,17-19). Therefore, the capability of USPIO to modulate the effective transverse relaxation rate, $\mathrm{R}_{2}{ }^{*}$, is exploited where the difference image of $\mathrm{R}_{2}{ }^{*}$ before and after administration of USPIO will be useful in determining microvessel density.

A gradient echo (GE) MRI sequence allows BV assessments in relatively shorter data acquisition times and has the advantage of minimizing artifacts associated with flow effects. A recent report with brain tumor model also shows that the $\mathrm{R}_{2}{ }^{*}$ is more sensitive to the USPIO induced susceptibility change (20) and can be used to estimate tumor BV. Although $\mathrm{BV}$ is linearly proportional to $\Delta \mathrm{R}_{2}$ and $\Delta \mathrm{R}_{2}{ }^{*}$, the proportionality constant was shown to be contrast agent dose-dependent $(13,16)$. The apparent BV values of tissues with different composition of arteries, capillaries and veins were shown to be different (13) since $\Delta R_{2}$ and $\Delta R_{2}{ }^{*}$ are affected by the vessel size $(12,18)$.

The design of the present study was to: a) optimize the choices of the MRI imaging parameters to evaluate the application of GE sequence; b) optimize USPIO dosage and TE based on $\mathrm{T}_{2}{ }^{*}$ changes with blood USPIO concentration after intravenous injection; and c) improve the calculations for the assessment of BV using the difference of pre- and postcontrast signals. Hypo-intense regions with very low proton density (e.g., bone and necrotic areas) in the pre-contrast image contribute to background noise leading to unreliable estimates of BV. A weighting scheme is proposed to BV values to suppress noise arising from such hypo-intense regions. The improved $\mathrm{BV}$ imaging technique was applied to evaluate tumor BV change response to anti-angiogenesis treatment in mice.

\section{Materials and methods}

Animals. Six to eight weeks old female $\mathrm{C} 3 \mathrm{H}$ mice weighing 20-30 g supplied by the Frederick Cancer Research Center, Animal Production (Frederick, MD) were used for in vivo and $e x$ vivo studies. The animals were housed in climate controlled and circadian rhythm-adjusted rooms and were fed ad libitum. Squamous cell carcinoma (SCCVII) cells were injected subcutaneously as a single suspension of $5 \times 10^{5}$ cells in the right hind leg. The tumors grew to $1.5 \mathrm{~cm}$ diameter $\left(1800 \mathrm{~mm}^{3}\right)$ in 10 days. All in vivo experiments were carried out in compliance with National Institutes of Health approved guidelines for animals [the Guide for the Care and Use of Laboratory Animal Resources (1996), National Research Council, and approved by the National Cancer Institute Animal Care and Use Committee].
Animal preparation for MRI. In SCC tumor study, mice were anesthetized by isoflurane $(1.5 \%)$ inhalation and mounted prone on a home built $25 \mathrm{~mm}$ (inner diameter) transmit receive resonator coil and holder ensemble where the coil axis (y-direction) was perpendicular to the main magnetic field. This specially designed holder enabled us, not only to mount the animal comfortably with its hip positioned downwards in the resonator while the upper body lay along the $\mathrm{z}$-axis, but also to place phantoms (fiducials). For brain imaging, a 35-mm birdcage coil was used for transmission and reception. A pressure transducer (SA Instruments, Inc., NY, USA) was placed on the mouse to monitor its breathing rate at $60 \pm 10$ per min under anesthesia. A non-magnetic rectal temperature probe (FISO, Quebec, Canada) was used to monitor the core body temperature of the mouse which was maintained at $36 \pm 1^{\circ} \mathrm{C}$ by a steady flow of warm air. A 30 gauge needle was cannulated into the tail vein and extended using optimum length of polythene tubing (PE-10) for administering USPIO (Molday ION $^{\mathrm{TM}}, 10 \mathrm{~g} \mathrm{Fe} / \mathrm{l}$, which can be converted to $179 \mathrm{mM}$ as concentration of Fe ion, colloidal size of $30 \mathrm{~nm}$, BioPAL Inc., Worcester, MA, USA). MRI studies were performed in a $7 \mathrm{~T}$ horizontal scanner operating on a Paravision platform (Bruker Biospin, Billerica, MA, USA). In anti-angiogenesis study, treatment with Sunitinib (50 mg/kg/day, LC Laboratories, Woburn, MA, USA) or methylcellulose/Tween-80 as control by oral gavage was initiated 10 days after tumor implantation, and MRI measurements were carried out before, 2 days, and 4 days after start of treatment.

Phantoms. The MRI signal intensity dependence with USPIO concentration was assessed using capillary tubes $(1.5 \mathrm{~mm}$ diameter $\times 50 \mathrm{~mm}$ length) filled with USPIO at Fe concentrations of $0.36,0.9,1.8,2.39,4.48$ and $8.95 \mathrm{mM}$ (dilutions in phosphate-buffered saline: $1 / 500,1 / 200,1 / 100,1 / 75,1 / 40$ and 1/20). Each capillary was subsequently placed inside $5 \mathrm{~mm}$ i.d. glass tubes filled with $1 \%$ agarose gel. The signal from the surrounding gel in GE image $[\mathrm{TE} / \mathrm{TR}=5.4 / 250 \mathrm{msec}$, Flip angle $(\mathrm{FA})=45^{\circ}, \mathrm{FOV}=32 \times 32 \mathrm{~mm}$ and in plane resolution $=125 \mu \mathrm{m}$ ] provided a distinct background for clear demarcation of capillary walls and the detection of hypointensity regions outside the capillaries due to susceptibility artifacts at high USPIO concentrations. The signal intensity vs. TE traces were used to determine $\mathrm{R}_{2}{ }^{*}$ values at each of these concentrations and $\mathrm{R}_{2}{ }^{*}$ of PBS was subtracted to obtain $\Delta \mathrm{R}_{2}{ }^{*}$ values.

Ex vivo experiment. Dose-dependent changes in $\mathrm{T}_{2}{ }^{*}$ were assessed by intravenous administration of USPIO into mice $(\mathrm{n}=4)$ at dosage of $143,215,287$, and $358 \mu \mathrm{mol}$ per $\mathrm{kg}$. Ten min after the injection, $\sim 500 \mu 1$ of blood was drawn from each animal into sample tubes containing heparin (10 $\mu \mathrm{l}$ of $100 \mathrm{U} / \mathrm{ml}$ ) to prevent blood from clotting. Blood drawn from a fifth mouse that did not receive USPIO was assumed to correspond to the $\mathrm{T}_{2}{ }^{*}$ value prior to USPIO administration. Glass tubes filled with these blood samples were placed vertically in the RF coil and GE-MR images were acquired at six different TE values ranging from 3.4 to $50 \mathrm{msec}$ (TR = $250 \mathrm{msec}, \mathrm{FA}=45^{\circ}$, in plane resolution $=125 \mu \mathrm{m}$, slice thickness $=2 \mathrm{~mm}, 3$ slices). $\mathrm{T}_{2}{ }^{*}$ maps were calculated by ImageJ software package (http://rsb.info.nih.gov/ij/) using MRI 
analysis calculator plug-in (Karl Schmidt, HypX Laboratory, Brigham and Women's Hospital).

In vivo MRI experiments. A set of orthogonally placed scout images through the tumor and normal leg were acquired using a fast spin echo (FSE) sequence (TE/TR $=47 / 2500 \mathrm{msec}$, inplane resolution $=250 \mu \mathrm{m}$, slice thickness $=2 \mathrm{~mm}, 8$ slices, 8 echoes). Using these pilot images, 16 contiguous slices encompassing the entire tumor was acquired using a flow compensated GE sequence $\left(\mathrm{TE} / \mathrm{TR}=5.4 / 250 \mathrm{msec}, \mathrm{FA}=45^{\circ}\right.$, in plane resolution $=125 \mu \mathrm{m}$, slice thickness $=2 \mathrm{~mm}, \mathrm{FOV}=$ $32 \times 32 \mathrm{~mm}$, number of acquisitions $=4$, total imaging time $\sim 4.5 \mathrm{~min}$ ). USPIO was infused in four increments from 143 to $358 \mu \mathrm{mol} / \mathrm{kg}$ in steps of $72 \mu \mathrm{mol} / \mathrm{kg}$ with $\sim 50 \mu 1$ of PBS flush following each infusion. Five min after each USPIO infusion and saline flush, which allowed USPIO to reach a steady state in vivo, post-USPIO acquisition was performed under identical scan parameters. Stereotaxically fixed tumor leg remained in alignment during pre- and post-USPIO scans. A capillary tube filled with water was used as a standard to correct for any signal variations between pre- and post-USPIO images. It was assumed that the blood USPIO level increased linearly by incremental infusions considering the long halflife of USPIO relative to the experiment time $(\sim 30 \mathrm{~min})$.

Improved blood volume calculation. In order to improve the $\mathrm{BV}$ calculation an expression was derived (Appendix for details derivation). In essence, this formulation is an extension of equation [1], but in contrast, takes into account possible experimental and theoretical limitations.

$\mathrm{BV}(\%)=100\left(1+\mathrm{D}_{\mathrm{f}}\right)\left(\mathrm{S}^{\text {pre }}-\mathrm{S}^{\text {post }}\right) /\left[\mathrm{S}^{\text {pre }}+\mathrm{S}^{\text {post }}\left(\mathrm{W}_{\mathrm{b}} / \mathrm{W}_{\mathrm{t}}-1\right)+\mathrm{D}_{\mathrm{f}}\right.$ $\left.\mathrm{S}_{\max }^{\mathrm{pre}}\right]$

where $S^{\text {pre }}$ and $S^{\text {post }}$ are the image intensities before and after USPIO infusion, $\mathrm{D}_{\mathrm{f}}$ is a numerical value chosen intuitively to account for image noise within an image. $\mathrm{W}_{\mathrm{b}}(\sim 0.9)$ and $\mathrm{W}_{\mathrm{t}}$ $(\sim 0.8)$ are volume fractions of intra- and extravascular water contents and is maximum signal intensity of $S^{\text {pre }}$ image.

\section{Results}

Phantom studies. Basic relationship between USPIO concentration, image intensity, and detrimental susceptibility artifact was investigated in vitro using MRI image of capillaries filled with different concentrations of USPIO in the range of 0-8.95 mM Fe (Fig. 1A). Mean pixel intensity from regions of interests (ROI) chosen inside the capillary area vs. USPIO concentration is shown in Fig. 1B. A linear correlation was observed between $\Delta \mathrm{R}_{2}{ }^{*}$ values and USPIO concentrations up to $4.48 \mathrm{mM}$ (Fig. 1C). Proportionality constant between $\mathrm{R}_{2}$ and USPIO concentration was reported earlier as $105.9 \pm 1.9 \mathrm{mM}^{-1}$ $\mathrm{sec}^{-1}$ in saline solutions (11). Results from our studies using GE sequence agree with this estimate for $\Delta \mathrm{R}_{2}{ }^{*} \mathrm{vs}$. Molday ION concentration dependence $\left(106 \pm 5 \mathrm{mM}^{-1} \mathrm{sec}^{-1}\right)$ as shown in the slope of the plot in Fig. 1C. Image intensity reduction can be observed outside the capillaries filled with more than $4.48 \mathrm{mM}$ USPIO with TE values longer than $5 \mathrm{msec}$ (Fig. 1A), which may cause overestimation of BV in vivo.
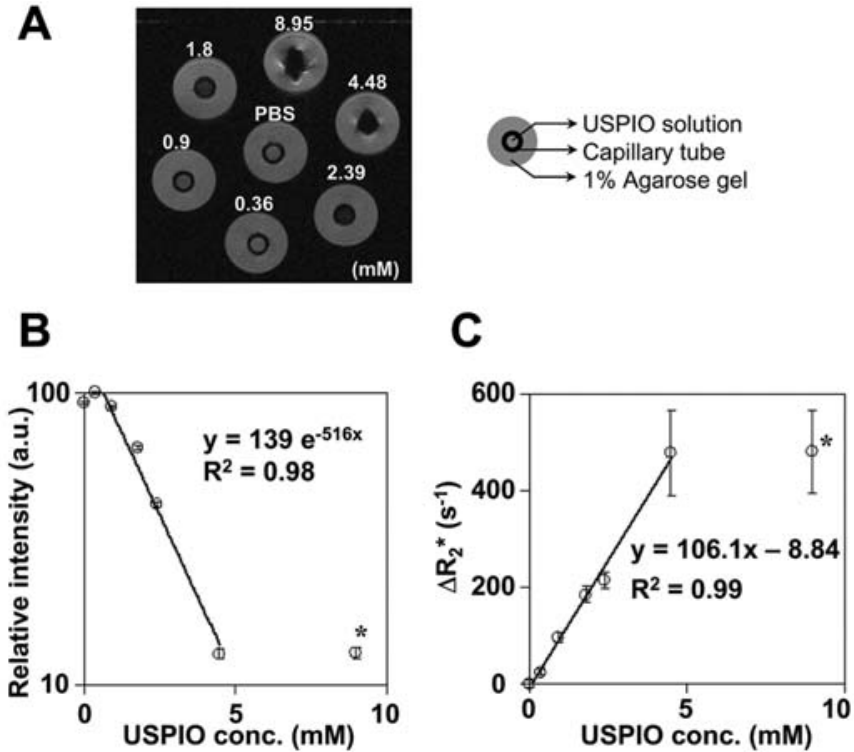

Figure 1. In vitro measurements using capillary tubes filled with PBS and USPIO at concentrations of $0.36,0.9,1.8,2.39,4.48$ and $8.95 \mathrm{mM} \mathrm{Fe}$ (dilutions of Molday ION ${ }^{\mathrm{TM}}$ in PBS: $1 / 500,1 / 200,1 / 100,1 / 75,1 / 40$ and $1 / 20$ ). (A), GE image showing the appearance of signal loss at $1.8 \mathrm{mM}$ and susceptibility artifact originating at $4.48 \mathrm{mM}$. (B), Relative intensities from each tube as a function of Fe concentration of USPIO. (C), $\left(\Delta \mathrm{R}_{2}{ }^{*}\right)$ vs. Fe concentration of USPIO. Slope $=106 \times 10^{3}\left( \pm 5 \times 10^{3}\right) \mathrm{s}^{-1} \mathrm{M}^{-1}$. The point marked by asterisk is inaccurate estimate resulting from signals approaching noise at USPIO concentration of 4.48 and above.

Ex vivo study. MRI images of tubes containing blood samples drawn from the animals that were administered different doses of USPIO shows signal intensity variation in a dose-dependent manner (Fig. 2A). At TE $=3.4 \mathrm{msec}$, the intensity reduction was over $98 \%$ at $287 \mu \mathrm{mol} / \mathrm{kg}$ (tube iii) and approached $100 \%$ at the higher dose (tube iv). At longer TE values ( $5 \mathrm{msec}$ ), $98 \%$ intensity reduction was achieved at lower USPIO concentration $(215 \mu \mathrm{mol} / \mathrm{kg})$. Therefore, at lower doses (143 and $215 \mu \mathrm{mol} / \mathrm{kg}$ ), the signal decay profile is sufficiently well characterized so that $\mathrm{T}_{2}{ }^{*}$ values from signal intensity vs. TE plots (Fig. 2B) can be reliably estimated. However, at higher doses $(287$ and $358 \mu \mathrm{mol} / \mathrm{kg}$ ), where the signal approached noise levels, in order to estimate $\mathrm{T}_{2}{ }^{*}$ it was necessary to extrapolate signal value at $\mathrm{TE}=0$ to define sufficient points on the decay curve. The $\mathrm{T}_{2}{ }^{*}$ values calculated are, respectively, $49,4.9,1.5,0.89,0.72 \mathrm{msec}$ for $0,143,215,287,358 \mu \mathrm{mol} / \mathrm{kg}$ USPIO doses (Fig. 2). The relationship between $\Delta \mathrm{R}_{2}{ }^{*}$ and USPIO dose was found to be non-linear in contrast to linear relationship observed in saline solutions (data not shown). The slope of this curve increases with the dose of USPIO which is attributed to clearance of USPIO from the blood pool in the animal. Simulated relative intensity differences ( Spre $^{\text {- }} \mathrm{S}^{\text {post }}$ ) vs. TE using above $\mathrm{T}_{2}{ }^{*}$ values are shown in Fig. 2D. The vertical line at $\mathrm{TE}=5 \mathrm{msec}$ indicates optimal value for the doses in the range of $215-287 \mu \mathrm{mol} / \mathrm{kg}$ where $S^{\text {post }}$ approaches noise level and $\left(\mathrm{S}^{\text {pre }}-\mathrm{S}^{\text {post }}\right)$ approaches maximum.

In vivo studies. Based on the data from saline solutions and blood samples, the optimum USPIO dosage was estimated to be between $215-287 \mu \mathrm{mol} / \mathrm{kg}$ and in vivo GE-MR images were acquired at $\mathrm{TE}=5.4 \mathrm{msec}$. An image (4th of 8 slices) of pre- 
A
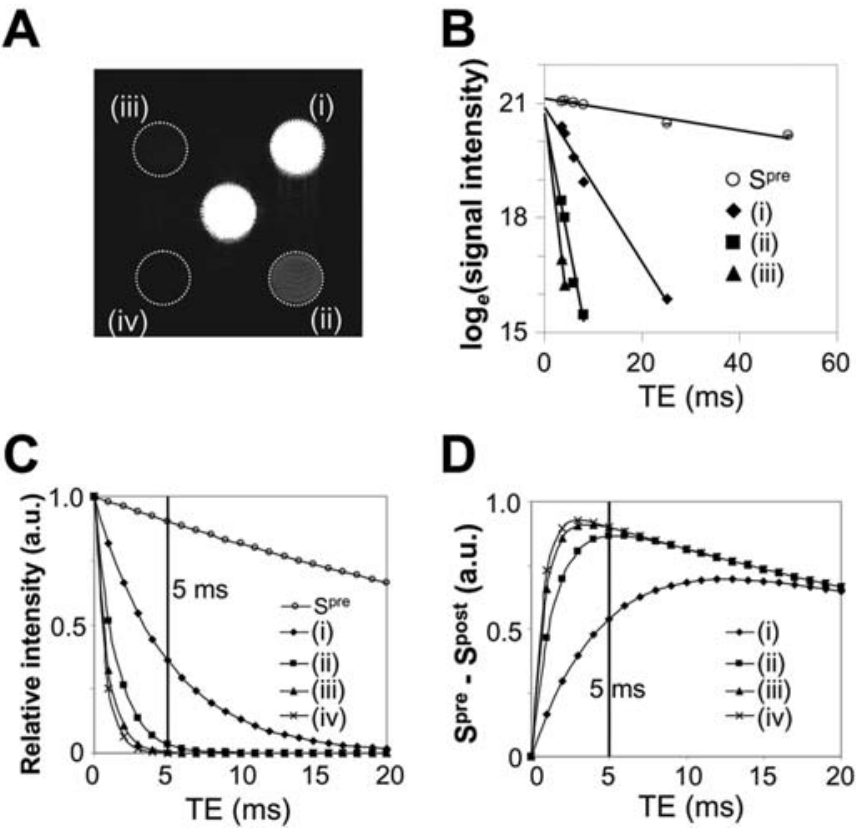

D

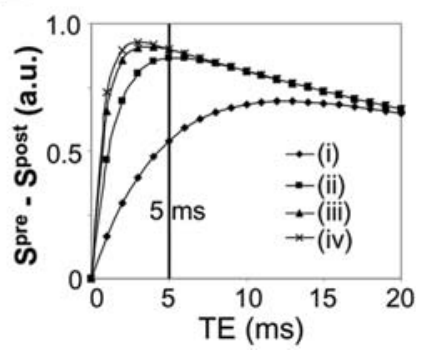

Figure 2. MR signal intensity variation with USPIO concentration in mouse blood (A) tubes filled with blood samples (inside dashed circles) with USPIO doses of (i) 143 , (ii) 215 , (iii) 287 and (iv) $358 \mu \mathrm{mol} / \mathrm{kg}$. The center image is from a tube containing normal blood drawn from a mouse that did not receive USPIO (normal). (B), Relative intensity variations by USPIO dose and TE. Spre is signal from normal mouse blood. Signals below background level were not shown. (C), Simulated intensity vs. TE curves at the doses (i)(iv) using $\mathrm{T}_{2}{ }^{*}$ values (i) 4.9 , (ii) 0.15 , (iii) 0.89 , (iv) $0.72 \mathrm{msec}$ respectively. $\mathrm{S}^{\text {pre }}$ values were calculated using $\mathrm{T}_{2}{ }^{*}=49 \mathrm{msec}$ obtained from center tube.

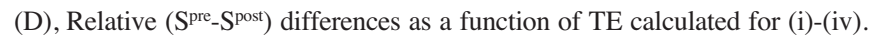
The vertical line drawn at $5 \mathrm{msec}$ indicates the optimal TE value for 215 $287 \mu \mathrm{mol} / \mathrm{kg}$ doses of USPIO where Spost approaches noise level and the

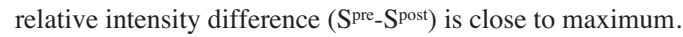

and post-USPIO images of a mouse brain is shown in Fig. 3A and $\mathrm{B}$.

As mentioned in Materials and methods, BV estimation using equation [1], is affected by regional variation of Spre, the pre-contrast MR intensity, in vivo. In contrast, for the proposed equation [2] and appendix the weighting of BV (\%) as a function of $S^{p r e}(\%)$ at different damping $\left(\mathrm{D}_{\mathrm{f}}\right)$ values are shown in Fig. 3C. The effect of $D_{f}$ on calculated BV image using equation [2], is shown in Fig. 3D-I. BV images were calculated from pre- and post-USPIO images (Fig. 3A and B) according to equation [2] using $\mathrm{D}_{\mathrm{f}}=0,0.02$, and 0.04 (Fig. 3D-F). The histograms of these images in the $\mathrm{BV}(\%)$ range of $0-100 \%$ are shown in Fig. 3G-I. The results shown in these figures indicate that unreliable estimation of BV resulting from the noise in background. Such hypo-intense regions of $\mathrm{S}^{\text {pre }}$ can be suppressed by the introduction of weighting factor $D_{f}$ into the basic BV calculation formula.

In vivo effect of the dose of USPIO was investigated in a normal mouse brain and a tumor implanted in the mouse leg. Average cerebral blood volume (CBV) in cortex region and tumor blood volume (TBV) were calculated at four different USPIO doses from 143 to $358 \mu \mathrm{mol} / \mathrm{kg}$ at $\mathrm{TE}=5.4 \mathrm{msec}$ using $\mathrm{D}_{\mathrm{f}}=0.02$ (Fig. 4). The calculated CBV for the four USPIO doses were between 1.5-17.3\%, while BVs calculated in the tumor region were between $16.7-44.3 \%$ at doses of 143, 215, 287 , and $358 \mu \mathrm{mol} / \mathrm{kg}$, respectively (Table I). Interestingly,
Table I. Average BV values of ROI in brain, tumor, and muscle as a function of USPIO dose calculated using equation [2].

\begin{tabular}{cccc}
\hline Dose $(\mu \mathrm{mol} / \mathrm{kg})$ & Brain $(\%)$ & Tumor $(\%)$ & Muscle (\%) \\
\hline 143 & 1.5 & 16.7 & 5.1 \\
215 & 5.9 & 26.4 & 6.1 \\
287 & 17.3 & 34.7 & 6.3 \\
358 & 15.4 & 44.3 & 7.8 \\
\hline
\end{tabular}

the CBV values gradually increased with dose and reached a plateau at $287 \mu \mathrm{mol} / \mathrm{kg}$ dose while there was a continuous increase in TBV for the four USPIO doses used. In addition, BV (\%) from muscle area of normal leg was assessed at each of these doses (Table I). These results suggest that the use of optimum USPIO dose and TE value are imperative to estimate the accurate $\mathrm{BV}$.

Anti-angiogenic drug studies. Anti-angiogenic treatment is an emerging strategy not only to suppress the tumor growth by shutting off the nutrients and oxygen supply to tumors but also to improve the efficiency of cytotoxic drug and radiotherapy via transit vascular normalization. Feasibility study of monitoring anti-angiogenic effect of Sunitinib, a multitargeted tyrosine kinase inhibitor under phase III clinical trial, was conducted and results are shown in Fig. 5. BV imaging was implemented with USPIO dose of $215 \mu \mathrm{mol} / \mathrm{kg}$ and $\mathrm{TE}=5.4 \mathrm{msec}$. The SCC tumor region shows high $\mathrm{BV}$ values $(24.3 \pm 12.5 \%, n=4)$ before anti-angiogenic treatment suggesting promoted angiogenesis in this tumor. It is noteworthy that in spite of such high BV values, this SCC tumor shows significant hypoxic fractions at this tumor size (4). Substantial decrease in the tumor BV was recognized in the BV images 2 and 4 days after start of daily Sunitinib administration $(15.0 \pm 3.3 \%$ and $13.1 \pm 8.8 \%$ at days 2 and 4 , respectively).

\section{Discussion}

USPIO has been used as a blood pool contrast agent to estimate tumor BV using MRI $(4,20)$ and the characteristics of USPIO are well documented in several reports (21-24). Steady state BV (\%) estimations using USPIO are affected by both external (its concentration, stability, and imaging parameters) and in vivo factors (time to equilibrate in the blood pool, half-life). Therefore, it is necessary to optimize parameters such as the dose, the time between the injection and the start of data acquisition to establish steady state, and the speed of data acquisition.

The USPIO used in this study is an iron oxide-based super paramagnetic contrast agent having a size of $30 \mathrm{~nm}$. It is a blood pool agent with a long intravascular half-life (21) causing susceptibility variations proximal to blood vessels making it suitable for $\mathrm{R}_{2}$ and $\mathrm{R}_{2}{ }^{*}$ measurements at steady state concentrations. A preliminary pharmacokinetic study (data not shown) indicated that USPIO reaches equilibrium levels in the blood of mice within 5 min after injection. USPIO has dominant $\mathrm{T}_{2}{ }^{*}$ effects over $\mathrm{T}_{1}$ unlike gadolinium agents which affect both $\mathrm{T}_{1}$ and $\mathrm{T}_{2}(21)$. 

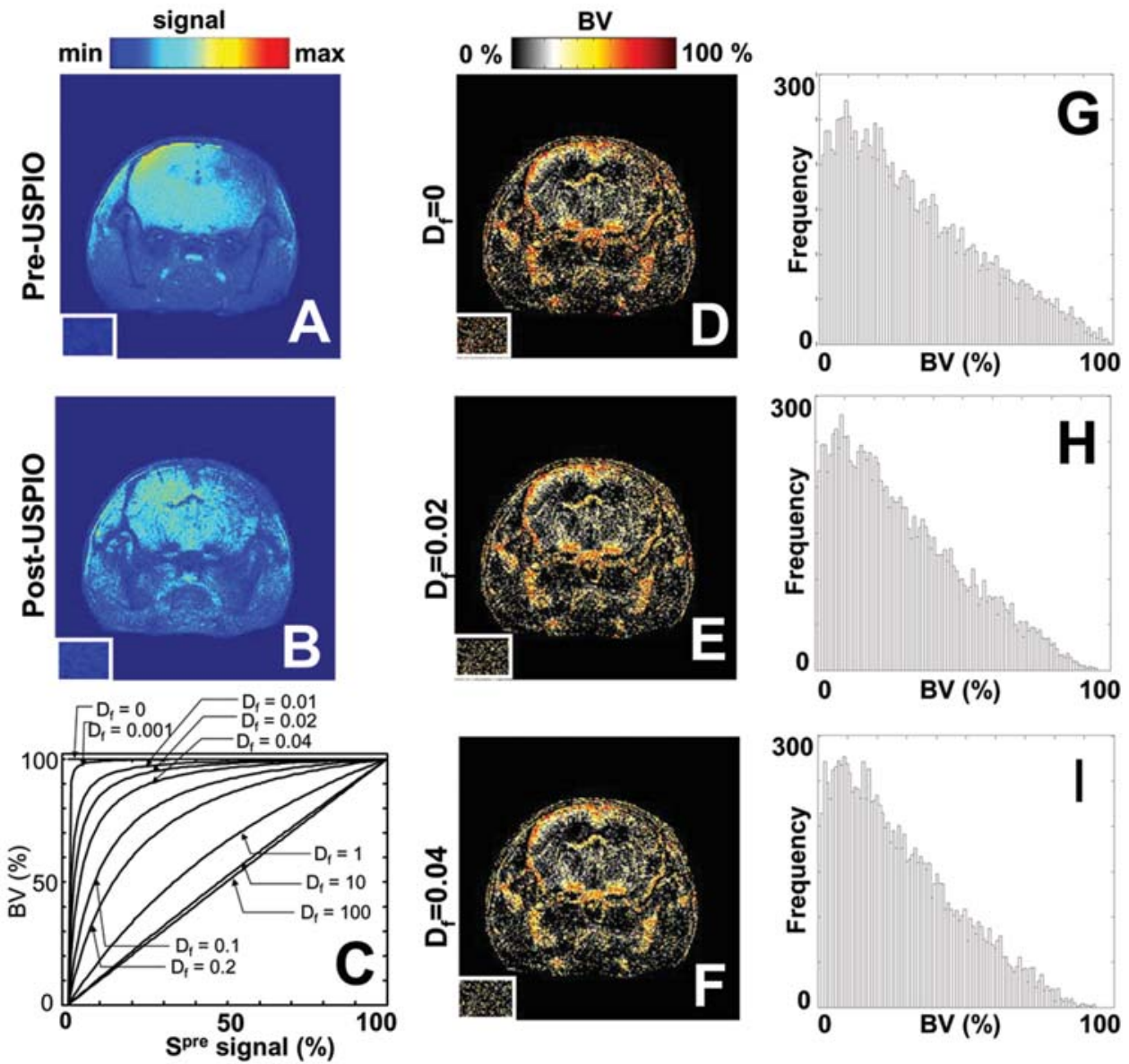

Figure 3. Effect of $\mathrm{D}_{\mathrm{f}}$ on BV (\%) calculation. (A and B), A MRI images of the mouse brain before and after USPIO administration (215 $\left.\mu \mathrm{mol} / \mathrm{kg}\right)$. (C), Weighting scheme for BV (\%) based on pre-USPIO signal ( $\left.\mathrm{S}^{\text {pre }}\right)$ at different values of $\mathrm{D}_{\mathrm{f}}$. At $\mathrm{D}_{\mathrm{f}}=0, \mathrm{BV}=100 \%$ remains unaffected by $\mathrm{S}^{\text {pre }}$. When $\mathrm{D}_{\mathrm{f}}>0, \mathrm{BV}$ $(\%)$ value is reduced depending on $\mathrm{S}^{\text {pre }}$ value. (D-F), BV (\%) images calculated at $\mathrm{D}_{\mathrm{f}}=0,0.02$ and 0.04 from equation [2]. The background is masked to accentuate the brain region but the inset at the bottom left show the original noise which is reduced as a function of $\mathrm{D}_{\mathrm{f}}$. High $\mathrm{BV}(\%)$ values in the background noise and the hypo-intense regions of Spre are attenuated at $\mathrm{D}_{\mathrm{f}}=0.04$. (G-I), Histograms of BV (\%) images at $\mathrm{D}_{\mathrm{f}}=0,0.02$ and 0.04 using BV (\%) $>0$ within the mouse region. The histograms of $\mathrm{BV}(\%)$ at $\mathrm{D}_{\mathrm{f}}=0.02$ and 0.04 show gradual increase in frequencies of low $\mathrm{BV}(\%)$. The contrast between the mouse region and background increases at high $\mathrm{D}_{\mathrm{f}}$ values.
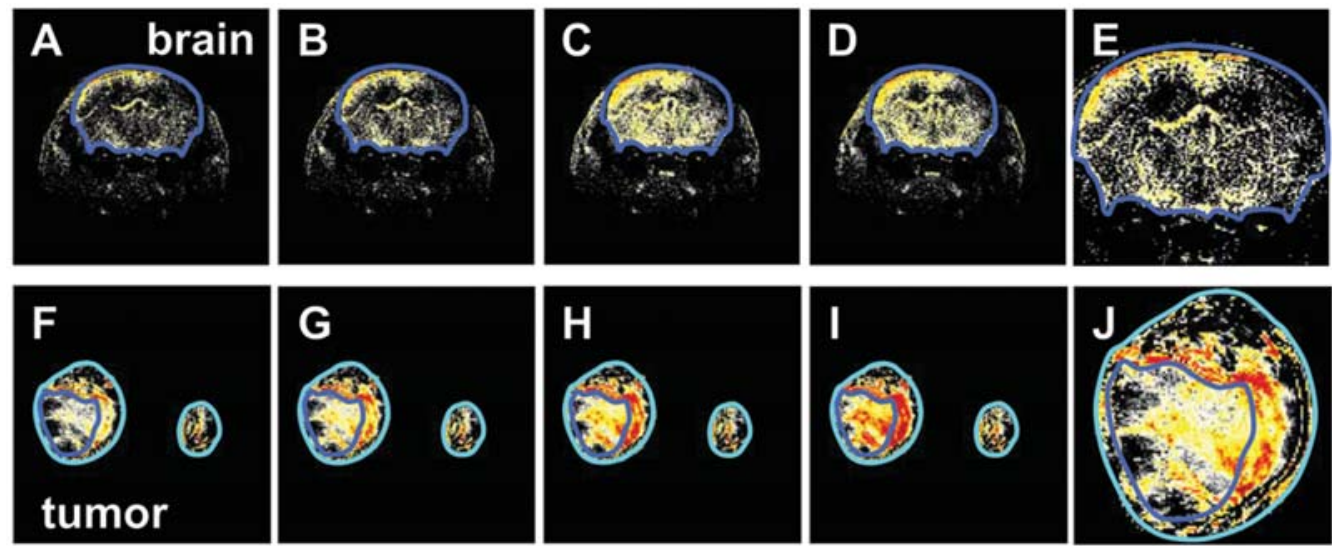

$143 \mu \mathrm{mol} / \mathrm{kg}$

$215 \mu \mathrm{mol} / \mathrm{kg}$

$287 \mu \mathrm{mol} / \mathrm{kg}$

$358 \mu \mathrm{mol} / \mathrm{kg}$

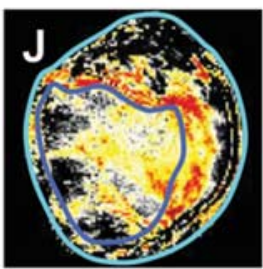

Blood Volume 0\% $100 \%$

Figure 4. (A-D), BV (\%) images (slice 4) of mouse brain at USPIO doses 143, 215, 287 and $358 \mu \mathrm{mol} / \mathrm{kg}$ calculated using equation [2] from GE-MR images. The ROI used for average brain BV (\%) calculation is enclosed by blue trace. (E), Enlarged view of ROI in (B). (F-I), Slice 4 of BV (\%) image of tumor bearing and normal legs of mouse at the same doses as in brain. The traces in cyan encompass both the mouse legs and ROI of the tumor in enclosed by blue trace. $(\mathrm{J})$, Enlarged view of tumor bearing leg in $\mathrm{g}$. TE/TR $=5.4 / 250 \mathrm{msec}$ FOV $=20 \times 20 \mathrm{~mm}$ for brain and $32 \times 32 \mathrm{~mm}$ for legs. Matrix size $=256 \times 256$ number of slices $=8$, slice thickness $=2 \mathrm{~mm}$, and averages $=2 . \mathrm{BV}(\%)$ images were calculated using $\mathrm{D}_{\mathrm{f}}=0.02$. 

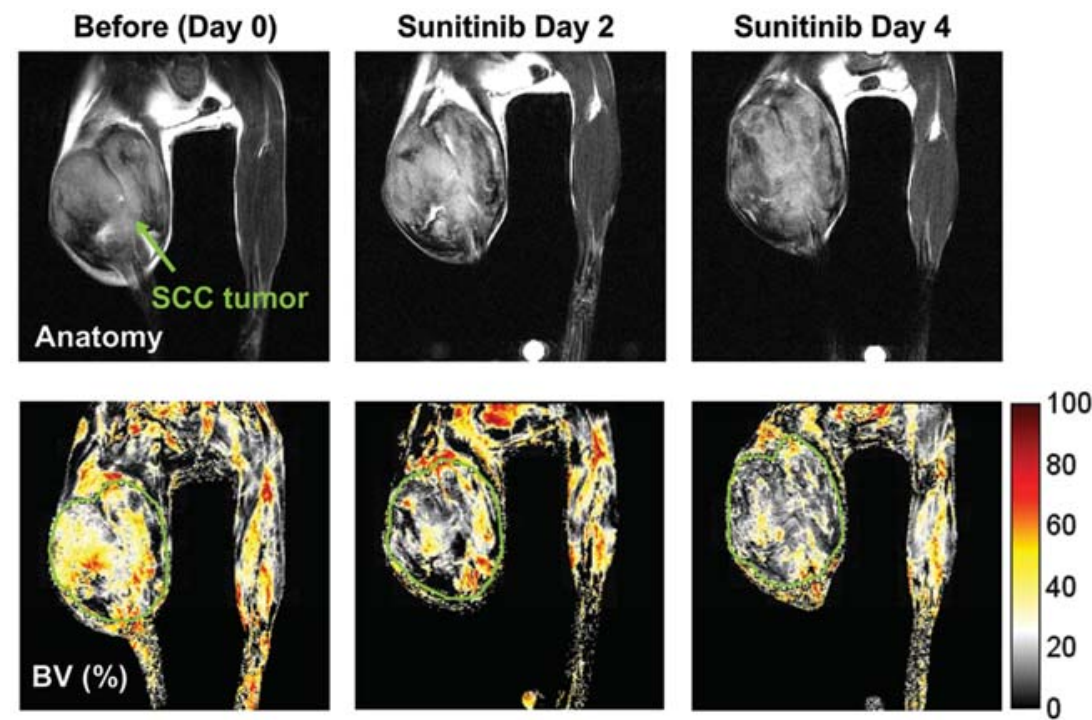

Figure 5. Effect of anti-angiogenic treatment on tumor blood volume. BV images of SCC tumor-bearing mice were obtained before, 2, and 4 days after start of daily Sunitinib treatment $(50 \mathrm{mg} / \mathrm{kg} /$ day $)$ with USPIO dose of $215 \mu \mathrm{mol} / \mathrm{kg}$ and TE $=5.4 \mathrm{msec}$.

The results of MRI studies in saline solutions containing USPIO, using GE sequence (Fig. $1 \mathrm{~B}$ and $\mathrm{C}$ ) on $\mathrm{R}_{2}{ }^{*}$ vs. USPIO concentration dependence agree with literature data (11). Deviation to the linear relationship of $\Delta \mathrm{R}_{2}{ }^{*}$ vs. dose was suggested by results from ex vivo studies. It is widely known that both $\Delta R_{2}{ }^{*}$ and $\Delta R_{2}$ are dependent on blood vessel size $(9,12,18)$, and the proportionality constant between $\Delta R_{2}{ }^{*}$ and $\mathrm{BV}(\%)$ is found to be dose and tissue dependent $(13,16)$. Therefore, $\Delta \mathrm{R}_{2}{ }^{*}$ and $\Delta \mathrm{R}_{2}$ for $\mathrm{BV}(\%)$ assessment using equations [1] or [2] (in this study) required scaling factors to obtain BV (\%) values which were found to correlate very well $(\mathrm{R}>0.995)$.

Optimum TE value. The difference ( $\mathrm{S}^{\text {pre }}$ - $\left.\mathrm{S}^{\text {post }}\right)$ in equation [2] depends on both the $\mathrm{T}_{2}{ }^{*}$ and $\mathrm{TE}$ values since the signal intensity of GE sequence is proportional to $\mathrm{e}^{-\left(\mathrm{TE} / \mathrm{T}_{2}\right)}$. The simulated relative intensity vs. TE curves using $\mathrm{T}_{2}{ }^{*}$ values derived from blood samples is shown in Fig. 2C. (Spre $\left.-S^{\text {post}}\right)$ vs. TE curves calculated for all four USPIO doses showed gradual increase of ( Spre $\left.^{\text {p }} \mathrm{S}^{\text {post }}\right)$ to a peak value and then a slow decrease (Fig. 2D). The signal intensity vs. TE curves (Fig. 2C) and $\left(S^{\text {pre }}-S^{\text {post }}\right)$ vs. TE curves (Fig. 2D) suggest that an optimal $\mathrm{TE}$ value lies at $\mathrm{TE}>2 \mathrm{TE}_{\max }$. In practice, the attainable $\mathrm{TE}$ is also limited by the hardware imposing constraints on minimum TE value.

Choice of $D_{f}$ value. $\mathrm{D}_{\mathrm{f}}$ is an adjustable parameter whose value depends on how much noise suppression is desired. As $D_{f}$ increases, from $D_{f}=0$ (no attenuation), the BV (\%) calculated using signals greater than $50 \%$ are marginally attenuated. However, signals below $10 \%$ are attenuated to a relatively larger extent. Since high $D_{f}$ value underestimates overall BV (\%), a small and constant $\mathrm{D}_{\mathrm{f}}$ value is desirable for valid comparisons among different $\mathrm{BV}$ images. The $\mathrm{BV}$ images calculated at $\mathrm{D}_{\mathrm{f}}=0,0.02$, and 0.04 (Fig. 3D-F) demonstrate noise reduction in the background areas and contrast enhancement at higher $D_{f}$ values. This is reflected in the histograms of the BV images as an increase in the frequencies of low BV (\%).

Optimum USPIO dose. Since BV in brain has been relatively well established $(14,25,26)$, dose dependency of USPIO was first investigated in the mouse brain using identical scan parameters and dosages as in tumor studies. The non-linear relationship of the signal intensity to USPIO dose (ex vivo studies on blood samples) indicate a more complex situation in live animals. The doses that were achieved over $98 \%$ of signal attenuation $(215-287 \mu \mathrm{mol} / \mathrm{kg})$ corresponded to 3.6$4.8 \mathrm{mM}$ USPIO in saline assuming that the total blood plasma volume of the mouse was $6 \%$ of its body weight (27). In phantoms, conspicuous susceptibility variations (Fig. 1A) were observed at $4.8 \mathrm{mM}$ USPIO and higher (TE $\geq 5 \mathrm{msec}$ ). The calculated CBV was $17.3 \%$ at this dose whereas the known CBV of mice is only about $5 \%(14,25,26)$. As tight junctions in the blood brain barrier are impermeable to USPIO it is unlikely such high values are realistic. Change in $\mathrm{R}_{2}{ }^{*}$ will vary upon vessel size because of its dependency on the local hematocrit in larger vessels vs. smaller capillaries and therefore, GE images are sensitive to micro and macrovasculature (18). A comparison of BV (\%) images at low and high doses reveals that the overestimation stems mainly from the effect of USPIO in the extravascular regions through magnetic susceptibility and water exchange between blood and tissue. The lower USPIO dose of $215 \mu \mathrm{mol} / \mathrm{kg}$ was concluded to be preferable for in vivo study.

The tumor data (Table I) indicate that the calculated TBV increase proportionately with USPIO dose in contrast to CBV (\%). Since, USPIO is a plasma marker and it is more suited for blood plasma volume (28) estimates, it was assumed that the tumor blood vessels prevent leakage of USPIO. Secondly, as blood consists of plasma and red blood cells where the volume of the latter depends on local hematocrit, the regional variations of hematocrit and the effects of the local hematocrit in the microvasculature (and therefore, on $\mathrm{CBV}$ ) were assumed to be negligible. 
It should be noted that, due to proton exchange, the presence of contrast agent in the vascular bed reduces $T_{1}$ in the tissue, resulting in a possible underestimation of $\Delta R_{2}$. However, due to the use of a long TR in this study $(250 \mathrm{msec})$, the effects of $\mathrm{T}_{1}$ changes can be assumed to be small and neglected. Therefore, it was also assumed that the $\mathrm{T}_{1}$ relaxation effects due to USPIO on TBV calculations was minimum because of the TE/TR employed.

This study utilized the same concept of using the steady state susceptibility contrast, exploiting the linear relationship between the change in $\mathrm{R}_{2}{ }^{*}$ and blood concentration of the USPIO (29), but with an improved equation, to calculate the $\mathrm{BV}(\%)$. Low concentrations of tissue blood USPIO is likely to lead to underestimation of relative BV (\%) due competing $\mathrm{T}_{1}$ effects with $\mathrm{T}_{2}{ }^{*}$ effects. Dose-dependent increase in TBV in contrast to $\mathrm{CBV}$ indicates regional variations in $\mathrm{BV}(\%)$ estimates. Structure and function of tumor neovasculature are architecturally chaotic and blood flow through them is irregular. Therefore, although USPIO is primarily an intravascular agent, vascular anomalies associated with tumor angiogenesis can render the rather large USPIO not confined to the vascular network (27). Nevertheless, 5 min post infusion is the optimum time for attaining equilibrium (data not shown).

In conclusion, blood plasma volume estimates can be quantitative in GE-MRI experiments at optimum concentration of USPIO and TE. In practice, several factors prevent administering optimal USPIO concentration in vivo. Further, the noise and artifacts in images introduce a definite amount of error to absolute BV estimates. Low dose of USPIO ( $215 \mu \mathrm{mol} /$ $\mathrm{kg}$ ) is suitable to identify relatively high blood volume regions while higher doses reveal low BV region at the cost of blurring. The difference between the optimized USPIO doses in vitro and in vivo studies points out the importance of dose optimization to obtain absolute blood volume. The calculated BV (\%) values are associated with noise that arises from the noise in pre- and post-USPIO images. The adjustable parameter $D_{f}$ in equation [2] reduces the noise in $\mathrm{BV}(\%)$ image improving its contrasts.

\section{References}

1. Brown JM and Giaccia AJ: The unique physiology of solid tumors: opportunities (and problems) for cancer therapy. Cancer Res 58: 1408-1416, 1998.

2. Naumov GN, Akslen LA and Folkman J: Role of angiogenesis in human tumor dormancy: animal models of the angiogenic switch. Cell Cycle 5: 1779-1787, 2006

3. Gatenby RA and Gillies RJ: Why do cancers have high aerobic glycolysis? Nat Rev Cancer 4: 891-899, 2004.

4. Matsumoto S, Hyodo F, Subramanian S, Devasahayam N, Munasinghe J, Hyodo E, Gadisetti C, Cook JA, Mitchell JB and Krishna MC: Low-field paramagnetic resonance imaging of tumor oxygenation and glycolytic activity in mice. J Clin Invest 118: 1965-1973, 2008

5. Jain RK: Normalizing tumor vasculature with anti-angiogenic therapy: a new paradigm for combination therapy. Nat Med 7: 987-989, 2001.

6. Bhattacharya A, Seshadri M, Oven SD, Toth K, Vaughan MM and Rustum YM: Tumor vascular maturation and improved drug delivery induced by methylselenocysteine leads to therapeutic synergy with anticancer drugs. Clin Cancer Res 14: 3926-3932, 2008.

7. Dings RP, Loren M, Heun H, McNiel E, Griffioen AW, Mayo KH and Griffin RJ: Scheduling of radiation with angiogenesis inhibitors anginex and Avastin improves therapeutic outcome via vessel normalization. Clin Cancer Res 13: 3395-3402, 2007.
8. Corot C, Robert P, Idee JM and Port M: Recent advances in iron oxide nanocrystal technology for medical imaging. Adv Drug Deliv Rev 58: 1471-1504, 2006.

9. Dennie J, Mandeville JB, Boxerman JL, Packard SD, Rosen BR and Weisskoff RM: NMR imaging of changes in vascular morphology due to tumor angiogenesis. Magn Reson Med 40: 793-799, 1998.

10. Enochs WS, Harsh G, Hochberg F and Weissleder R: Improved delineation of human brain tumors on MR images using a longcirculating, superparamagnetic iron oxide agent. J Magn Reson Imaging 9: 228-232, 1999 .

11. Gambarota G, van Laarhoven HW, Philippens M, Lok J, van der Kogel A, Punt CJ and Heerschap A: Assessment of absolute blood volume in carcinoma by USPIO contrast-enhanced MRI. Magn Reson Imaging 24: 279-286, 2006.

12. Kiselev VG, Strecker R, Ziyeh S, Speck O and Hennig J: Vessel size imaging in humans. Magn Reson Med 53: 553-563, 2005.

13. Pathak AP, Rand SD and Schmainda KM: The effect of brain tumor angiogenesis on the in vivo relationship between the gradient-echo relaxation rate change (DeltaR2*) and contrast agent (MION) dose. J Magn Reson Imaging 18: 397-403, 2003.

14. Persigehl T, Bieker R, Matuszewski L, Wall A, Kessler T, Kooijman H, Meier N, Ebert W, Berdel WE, Heindel W, Mesters RM and Bremer C: Antiangiogenic tumor treatment: early noninvasive monitoring with USPIO-enhanced MR imaging in mice. Radiology 244: 449-456, 2007.

15. Tropres I, Grimault S, Vaeth A, Grillon E, Julien C, Payen JF, Lamalle L and Decorps M: Vessel size imaging. Magn Reson Med 45: 397-408, 2001

16. Van Osch MJ, Vonken EJ, Viergever MA, van der Grond J and Bakker CJ: Measuring the arterial input function with gradient echo sequences. Magn Reson Med 49: 1067-1076, 2003.

17. Belliveau JW, Rosen BR, Kantor HL, Rzedzian RR, Kennedy DN, McKinstry RC, Vevea JM, Cohen MS, Pykett IL and Brady TJ: Functional cerebral imaging by susceptibility-contrast NMR. Magn Reson Med 14: 538-546, 1990.

18. Boxerman JL, Hamberg LM, Rosen BR and Weisskoff RM: MR contrast due to intravascular magnetic susceptibility perturbations. Magn Reson Med 34: 555-566, 1995.

19. Ostergaard L, Smith DF, Vestergaard-Poulsen P, Hansen SB, Gee AD, Gjedde A and Gyldensted C: Absolute cerebral blood flow and blood volume measured by magnetic resonance imaging bolus tracking: comparison with positron emission tomography values. J Cereb Blood Flow Metab 18: 425-432, 1998.

20. Gambarota G, Leenders W, Maass C, Wesseling P, van der Kogel B, van Tellingen $\mathrm{O}$ and Heerschap A: Characterisation of tumour vasculature in mouse brain by USPIO contrast-enhanced MRI. Br J Cancer 98: 1784-1789, 2008.

21. De Lussanet QG, Backes WH, Griffioen AW, van Engelshoven JM and Beets-Tan RG: Gadopentetate dimeglumine versus ultrasmall superparamagnetic iron oxide for dynamic contrast-enhanced MR imaging of tumor angiogenesis in human colon carcinoma in mice. Radiology 229: 429-438, 2003.

22. Shen T, Weissleder R, Papisov M, Bogdanov A Jr and Brady TJ: Monocrystalline iron oxide nanocompounds (MION): physicochemical properties. Magn Reson Med 29: 599-604, 1993.

23. Jung CW and Jacobs P: Physical and chemical properties of superparamagnetic iron oxide MR contrast agents: ferumoxides, ferumoxtran, ferumoxsil. Magn Reson Imaging 13: 661-674, 1995.

24. Le Duc G, van der Elst L, Colet JM, Roch A, Gillis P, Le Bas JF and Muller RN: Ultrasmall particulate iron oxides as contrast agents for magnetic resonance spectroscopy: a dose-effect study. J Magn Reson Imaging 13: 619-626, 2001.

25. Mathew NT, Meyer JS, Bell RL, Johnson PC and Neblett CR: Regional cerebral blood flow and blood volume measured with the gamma camera. Neuroradiology 4: 133-140, 1972.

26. Rostrup E, Knudsen GM, Law I, Holm S, Larsson HB and Paulson OB: The relationship between cerebral blood flow and volume in humans. Neuroimage 24: 1-11, 2005

27. Riches AC, Sharp JG, Thomas DB and Smith SV: Blood volume determination in the mouse. J Physiol 228: 279-284, 1973.

28. Le Duc G, Peoc'h M, Remy C, Charpy O, Muller RN, Le Bas JF and Decorps M: Use of T(2)-weighted susceptibility contrast MRI for mapping the blood volume in the glioma-bearing rat brain. Magn Reson Med 42: 754-761, 1999.

29. Barbier EL, Lamalle L and Decorps M: Methodology of brain perfusion imaging. J Magn Reson Imaging 13: 496-520, 2001. 


\section{Appendix}

Blood volume calculation. $\mathrm{BV}$ in a voxel is given by the ratio of volume of blood in the voxel to the tissue mass of the voxel and expressed in milliliters per 100 gram of tissue or microliters per gram $(\mu \mathrm{l} / \mathrm{g})$. Assuming that the total volume of the voxel can be divided by the volumes of intra- and extravascular compartments, $\mathrm{V}_{\mathrm{b}}$ and $\mathrm{V}_{\mathrm{t}}$ respectively, the $\mathrm{BV}(\%)$ can be defined as a volume fraction by (29),

BV $(\%)=100$ [volume of blood in a voxel/volume of the voxel] or

$$
\mathrm{BV}(\%)=100 \frac{\mathrm{V}_{\mathrm{b}}}{\mathrm{V}_{\mathrm{b}}+\mathrm{V}_{\mathrm{t}}}
$$

This model assume, one, that only the intravascular protons become MRI invisible through the magnetic susceptibility gradients caused by USPIO and ignores any susceptibility effects in the extravascular regions through water exchange between blood and tissue, two, the leakage of USPIO from disrupted blood vessels, and, three, $\mathrm{T}_{1}$ effects caused by USPIO. The signal from a voxel can be divided into two components: a) a component that comes from intravascular protons which is affected by contrast agent and b) a component from extravascular protons that is unaffected by contrast agent. When the signal from intravascular protons is effectively removed, Spost is proportional to extravascular volume. Therefore,

$$
\mathrm{S}^{\text {post }}=k \mathrm{~W}_{\mathrm{t}} \mathrm{V}_{\mathrm{t}}
$$

and the intravascular volume is,

$$
\left(\mathrm{S}^{\text {pre }}-\mathrm{S}^{\text {post }}\right)=k \mathrm{~W}_{\mathrm{b}} \mathrm{V}_{\mathrm{b}}
$$

where $k$ is a proportionality constant between signal and the number of protons and intra- and extravascular water fractions are $\mathrm{W}_{\mathrm{b}}(\approx 0.9)$ and $\mathrm{W}_{\mathrm{t}}(\approx 0.8)$ respectively $(11)$. Assume that $x$ is a fraction of signal that remains after this effect and $x=\mathrm{e}^{-\Delta \mathrm{R}_{2}{ }^{*} \mathrm{TE}}$, where $\Delta \mathrm{R}_{2}{ }^{*}=\frac{1}{\mathrm{~T}_{2}{ }^{*} \text { (USPIO) }}-\frac{1}{\mathrm{~T}_{2}{ }^{*}}$. The signals observed before $\left(\mathrm{S}^{\text {pre }}\right)$ and after ( $\left.\mathrm{S}^{\text {post}}\right)$ USPIO administration in the animal are therefore,

$$
\begin{aligned}
\mathrm{S}^{\text {pre }} & =\mathrm{S}_{\mathrm{b}}+\mathrm{S}_{\mathrm{t}} \\
\mathrm{S}^{\text {post }} & =x \mathrm{~S}_{\mathrm{b}}+\mathrm{S}_{\mathrm{t}}
\end{aligned}
$$

and the difference

$$
\mathrm{S}^{\text {pre }}-\mathrm{S}^{\text {post }}=(1-x) \mathrm{S}_{\mathrm{b}}
$$

Substituting $\Delta S=\left(S^{\text {pre }}-S^{\text {post }}\right)$, and $\delta=(1-x)$, and rearranging [A7],

$$
\mathrm{S}_{\mathrm{b}}=\Delta \mathrm{S} / \delta
$$

Rearranging equations [A2], [A3] and [A4] to extract $\mathrm{V}_{\mathrm{b}}, \mathrm{V}_{\mathrm{t}}$ and $\mathrm{S}_{\mathrm{t}}$ and substitution in [A1], lead to

$$
\mathrm{BV}(\%)=100 \frac{\Delta \mathrm{S} /\left(k \mathrm{~W}_{\mathrm{b}} \delta\right)}{\Delta \mathrm{S} /\left(k \mathrm{~W}_{\mathrm{b}} \delta\right)+\left(\mathrm{S}^{\text {pre }}-\Delta \mathrm{S} / \delta\right) /\left(k \mathrm{~W}_{\mathrm{t}}\right)}
$$

Multiplying the numerator and denominator by $k \mathrm{~W}_{\mathrm{b}} \delta,[\mathrm{A} 8]$ is simplified to

$$
\mathrm{BV}(\%)=100 \frac{\Delta \mathrm{S}}{\Delta \mathrm{S}+\left(\mathrm{W}_{\mathrm{b}} / \mathrm{W}_{\mathrm{t}}\right)\left(\delta \mathrm{S}^{\text {pre }}-\Delta \mathrm{S}\right)}
$$

Expansion of $\Delta S$ and $\delta$ in [A9] and rearrangement gives,

$$
\operatorname{BV}(\%)=100 \frac{\left(\mathrm{S}^{\text {pre }}-\mathrm{S}^{\text {post }}\right)}{\mathrm{S}^{\text {pre }}\left[1-x\left(\mathrm{~W}_{\mathrm{b}} / \mathrm{W}_{\mathrm{t}}\right)\right]+\mathrm{S}^{\text {post }}\left[\left(\mathrm{W}_{\mathrm{b}} / \mathrm{W}_{\mathrm{t}}\right)-1\right]}[\mathrm{A}
$$

When $x$ is negligibly small, equation [A10] can be simplified to

$$
\mathrm{BV}(\%)=100 \frac{\left(\mathrm{S}^{\text {pre }}-\mathrm{S}^{\text {post }}\right)}{\mathrm{S}^{\text {pre }}+\mathrm{S}^{\text {post }}\left[\left(\mathrm{W}_{\mathrm{b}} / \mathrm{W}_{\mathrm{t}}\right)-1\right]}
$$

The factor $\left(\mathrm{W}_{\mathrm{b}} / \mathrm{W}_{\mathrm{t}}-1\right)$ in the denominator accounts for the difference in intra- and extravascular water content. In hypointense regions where $S^{\text {pre }}=S^{\text {post }}=0$, the denominator in right hand side of equation [A11] would become zero. In this case, $\mathrm{BV}=0$ since $\mathrm{S}^{\text {pre }}$ must be greater than zero if $\mathrm{BV}>0$. In practice, unreliable and random BV estimates are obtained in these regions due to noise which can be suppressed by adding a small positive quantity $\sigma_{\mathrm{b}}$ (background noise) to the denominator in right hand side of equation [A11]. Defining a fraction $D_{f}$ such that $D_{f}=\sigma_{b} / S_{\max }^{\text {pre }}$ where $S_{\max }^{\text {pre }}$ is highest signal intensity of Spre image and adding this factor to denominator and rescaling by $\left(1+\mathrm{D}_{\mathrm{f}}\right)$, equation [11] becomes,

$\mathrm{BV}(\%)=100\left(1+\mathrm{D}_{\mathrm{f}}\right)\left(\mathrm{S}^{\text {pre }}-\mathrm{S}^{\text {post }}\right) /\left[\mathrm{S}^{\text {pre }}+\mathrm{S}^{\text {post }}\left(\mathrm{W}_{\mathrm{b}} / \mathrm{W}_{\mathrm{t}}-1\right)+\mathrm{D}_{\mathrm{f}}\right.$ $\left.\mathrm{S}_{\max }^{\text {pre }}\right]$

The limits of BV can be set between 0 and 100 for visual representations to eliminate unrealistic negative values arising from noise or increased $S^{\text {post }}$ intensities due to possible $\mathrm{T}_{1}$ effects. However, average BV (\%) estimates of a region of interest (ROI) are calculated without imposing these limits to account for random noise.

Choice of optimum TE and $D_{f}$ values. The signal intensity is the same for all USPIO concentrations at TE $=0$ but decays differently at different concentrations according to the $\mathrm{T}_{2}{ }^{*}$ values. The term (Spre $\left.-S^{\text {post}}\right)$ in equations [1] and [2] can be evaluated at each TE value since the samples with and without USPIO represent post- and pre-USPIO conditions. The peak occurs at a TE value,

$$
\mathrm{TE}_{\max }=\frac{\mathrm{T}_{2}^{* \text { pre }} \mathrm{T}_{2}^{* \text { post }}}{\mathrm{T}_{2}^{*} \text { pre }-\mathrm{T}_{2}^{* \text { post }}} \ln \left(\frac{\mathrm{T}_{2}^{* \text { pre }}}{\mathrm{T}_{2}^{* \text { post }}}\right)
$$

where $\mathrm{T}_{2}{ }^{*}$ pre and $\mathrm{T}_{2}{ }^{*}$ post are the $\mathrm{T}_{2}{ }^{*}$ values before and after USPIO administration. Although the peak ( $\left.S^{\text {pre }}-S^{\text {post }}\right)$ has the advantage of sensitivity, equation [2] also requires negligibly small value of Spost for reliable BV (\%) estimation. High Spre value is desirable for better SNR.

The ratio of $\sigma_{\mathrm{b}} / \mathrm{S}_{\max }^{\text {pre }}$ where $\sigma_{\mathrm{b}}$ is noise level that can be estimated by the standard deviation of background pixel intensities. For a ratio of $\sigma_{b} / \mathrm{S}_{\max }^{\text {pre }}=0.02, \mathrm{D}_{\mathrm{f}}$ can be between 0.02 and 0.04 . The weights tend to become linearly proportional to Spre when $D_{f} \gg>1$. 